\title{
Water Wave Scattering by Partially Reflecting Breakwaters
}

\author{
By Kyung-Duck Suh ${ }^{1}$ and Hanna Kim ${ }^{2}$
}

\begin{abstract}
Following the approach used by Penney and Price in 1952, analytical solutions are derived for water wave scattering by a semi-infinite breakwater or a breakwater gap of partial reflection. The water depth is constant and a regular wave train is normally incident to the breakwater. Wave scattering is studied based on the linear potential wave theory. The governing equation is transformed into ordinary differential equations by using the method of variation of parameters and coordinate transformation. Using the analytic solution, the tranquility of harbor entrance is investigated by changing the reflection coefficient at the breakwater. As expected, the wave height is reduced at the harbor entrance as the wave reflection from the breakwater decreases.
\end{abstract}

Keywords: Analytic solution; Breakwaters; Harbor tranquility; Wave diffraction; Wave reflection

\section{Introduction}

Numerous numerical models have been developed that predict the transformation of waves from the deep ocean to the coast. Since numerical models inherently involve approximations, it is necessary to test these models against both analytic solutions and laboratory and field data from representative cases. In theory, the most rigorous test cases would involve comparisons with laboratory and field data, because they are the physical systems of interest. However, such comparisons can be problematic, since it is difficult to measure or reproduce all the necessary boundary and forcing conditions in the experiments. Also, experimental data always contain a certain amount of measurement errors. Analytic solutions are another avenue for testing numerical models at reduced cost, time, and labor in comparison to experiments.

Wave scattering by semi-infinitely long breakwaters has long been a subject of coastal engineering researchers. Penney and Price (1952) proposed an analytic solution for diffracted

\footnotetext{
${ }^{1}$ Member, Professor, Department of Civil and Environmental Engineering \& Engineering Research Institute, Seoul National University, Seoul 151-744, Korea (E-mail: kdsuh@snu.ac.kr)

2 Researcher, Coastal Engineering Research Department, Korea Ocean Research \& Development Institute, Ansan P.O. Box 29, 425-600, Korea (E-Mail: sarah314@kordi.re.kr)
} 
waves around a semi-infinitely long impermeable breakwater based on Sommerfeld's (1896) solution for diffraction of light. They also obtained the solution for the waves transmitted through a gap in a breakwater by superposing the solutions for the semi-infinite breakwaters. Recently, Yu (1995) derived the boundary condition for a thin porous wall based on the formulation of Sollitt and Cross (1972), and used it to find an approximate solution for diffraction of water waves normally incident to a semi-infinite porous breakwater. More recently, McIver (1999) extended the analytic solution to obliquely incident waves using the Wiener-Hopf technique.

The solution of Penney and Price (1952) could be used for a vertical caisson breakwater, whilst those of Yu (1995) and McIver (1999) could be used for a rubble mound breakwater or any other porous breakwaters such as curtain wall or pile breakwaters. Nowadays, to reduce wave reflection from and impulsive wave pressure acting on a vertical caisson breakwater, a horizontally composite breakwater (i.e., a vertical caisson breakwater covered with wave-energydissipating concrete blocks) or a perforated-wall caisson breakwater is often used, which has a partially reflective front and solid back. Such type of breakwaters can also improve the conditions for vessel navigation in harbor entrance area, resulting in a safer approach to a harbor entrance or maneuvering within the entrance itself (see McBride et al., 1994). In the present study, following the approach of Penney and Price (1952), we derive analytic solutions for waves scattered by a semi-infinitely long breakwater of partial reflection. The solutions for the waves in the vicinity of a breakwater gap are then obtained by superposition from the solutions for the semi-infinite breakwater already considered. Finally, the derived solutions are used to test the tranquility of harbor entrance by changing the reflection coefficient at the front face of the breakwater.

\section{Analytic Solutions}

\subsection{Semi-infinite breakwater}

The plan view of a semi-infinitely long breakwater and the associated coordinate system are shown in Fig. 1. Cartesian coordinates $x, y$, and $z$ are chosen with origin by the mean free surface at the tip of the breakwater, the $x$-and $y$-axes lie in a horizontal plane and the $z$-axis is directed vertically upward. The water depth is constant as $h$, and the breakwater is placed along the positive $x$-axis. A regular wave train is normally incident to the breakwater from the negative infinity in the $y$-direction. 
Assuming incompressible fluid and irrotational flow motion, the velocity potential exists, which satisfies the Laplace equation. Linearizing the free-surface boundary conditions, the following boundary value problem for the velocity potential $\Phi(x, y, z, t)$ is obtained.

$$
\begin{aligned}
& \frac{\partial^{2} \Phi}{\partial x^{2}}+\frac{\partial^{2} \Phi}{\partial y^{2}}+\frac{\partial^{2} \Phi}{\partial z^{2}}=0 \\
& \frac{\partial \Phi}{\partial z}=0 \quad \text { at } z=-h \\
& -\frac{\partial \Phi}{\partial z}=\frac{\partial \eta}{\partial t} \quad \text { at } z=0 \\
& -\frac{\partial \Phi}{\partial t}+g \eta=0 \quad \text { at } z=0
\end{aligned}
$$

The velocity potential satisfying the no-flow bottom boundary condition is represented by

$$
\Phi(x, y, z, t)=A \cosh (z+h) F(x, y) e^{i \sigma t}
$$

where $k$ and $\sigma$ are the wave number and wave angular frequency, respectively, and $F(x, y)$ is a complex function. Substituting Eq. (5) into the Laplace equation yields the Helmholtz equation in $F(x, y)$ :

$$
\frac{\partial^{2} F}{\partial x^{2}}+\frac{\partial^{2} F}{\partial y^{2}}+k^{2} F(x, y)=0
$$

To solve this equation, we closely follow the approach of Sommerfeld (1896), which is also summarized in Lamb (1945, p. 538). The readers could also refer to Kim (2007) for more detailed procedure. The general solution to the preceding equation can be expressed as the sum of two solutions:

$$
F(x, y)=e^{-i k} F_{1}(x, y)+e^{i k} F_{2}(x, y)
$$

Since the procedure for solving the equation is the same for both solutions, the procedure is 
described only for one solution. Substituting the first solution into Eq. (6) gives

$$
\frac{\partial^{2} F_{1}}{\partial x^{2}}+\frac{\partial^{2} F_{1}}{\partial y^{2}}-2 i k \frac{\partial F_{1}}{\partial y}=0
$$

It is convenient to introduce the following parameters

$$
\begin{aligned}
& k x=\xi^{2}-\psi^{2}, \quad k y=2 \xi \psi \\
& k r=\xi^{2}+\psi^{2}
\end{aligned}
$$

where $r$ is the distance from the origin. We easily find

$$
\begin{aligned}
& \frac{\partial \xi}{\partial y}=\frac{\psi}{2 r}, \quad \frac{\partial \psi}{\partial y}=\frac{\xi}{2 r} \\
& \frac{\partial \xi}{\partial x}=\frac{\xi}{2 r}, \quad \frac{\partial \psi}{\partial x}=-\frac{\psi}{2 r} \\
& \frac{\partial^{2} \xi}{\partial x^{2}}+\frac{\partial^{2} \xi}{\partial y^{2}}=0, \quad \frac{\partial^{2} \psi}{\partial x^{2}}+\frac{\partial^{2} \psi}{\partial y^{2}}=0
\end{aligned}
$$

Using these relations, Eq. (8) can be expressed as an equation of $\xi$ and $\psi$ :

$$
\frac{\partial^{2} F_{1}}{\partial \xi^{2}}+\frac{\partial^{2} F_{1}}{\partial \psi^{2}}-4 i\left(\psi \frac{\partial F_{1}}{\partial \xi}+\xi \frac{\partial F_{1}}{\partial \psi}\right)=0
$$

This equation can be transformed into an ordinary differential equation of a single variable $\rho$ by using the relation $u=f(\xi-\psi)=f(\rho)$ :

$$
\frac{d^{2} f}{d \rho^{2}}+2 i \rho \frac{d f}{d \rho}=0
$$

Solving this equation, the following solution can be obtained:

$$
F_{1}=\alpha+\beta \int_{0}^{\xi-\psi} e^{-i \rho^{2}} d \rho
$$


Similarly, the second solution in Eq. (7) can be obtained as

$$
F_{2}=\gamma+\delta \int_{0}^{\xi+\psi} e^{-i \rho^{2}} d \rho
$$

The unknowns $\alpha, \beta, \gamma$, and $\delta$ can be obtained by applying the partial reflection condition in front of the breakwater $\left(y=0^{-}\right)$and the perfect reflection condition behind the breakwater $\left(y=0^{+}\right)$, i.e.

$$
\begin{aligned}
& \frac{\partial F(x, y)}{\partial x}+b F(x, y)=0 \quad \text { at } y=0^{-}, x \geq 0 \\
& \frac{\partial F(x, y)}{\partial x}=0 \text { at } y=0^{+}, x \geq 0
\end{aligned}
$$

The coefficient $b$ in Eq. (18) is complex, i.e., $b=b_{1}+i b_{2}$. Assuming that there is no phase difference between incident and reflected waves, we have

$$
b_{1}=0, b_{2}=k \cos \theta \frac{1-C_{r}}{1+C_{r}}
$$

where $C_{r}$ is the reflection coefficient at the breakwater, and $\theta$ is the incident wave angle. In the case of perfect reflection, $C_{r}=1$ so that $b=0$, which yields Eq. (19). Using the boundary conditions, Eqs. (18) and (19), the unknowns $\alpha, \beta, \gamma$, and $\delta$ are obtained as

$$
\begin{aligned}
& \alpha=\frac{1}{2}, \quad \beta=\frac{e^{\frac{1}{4} i \pi}}{\sqrt{\pi}}, \quad \gamma=\frac{C_{r}}{2}, \delta=\frac{C_{r} e^{\frac{1}{4} i \pi}}{\sqrt{\pi}} \quad \text { at } y<0 \\
& \alpha=\frac{1}{2}, \quad \beta=\frac{e^{\frac{1}{4} i \pi}}{\sqrt{\pi}}, \quad \gamma=\frac{1}{2}, \delta=\frac{e^{\frac{1}{4} i \pi}}{\sqrt{\pi}} \quad \text { at } \quad y>0
\end{aligned}
$$

Substitution of Eqs. (16) and (17) with these coefficients into Eq. (7) gives 


$$
F(x, y)= \begin{cases}\frac{1}{2}(1+i)\left(e^{-i k y} \int_{-\infty}^{\sigma} e^{-\frac{\pi}{2} i u^{2}} d u+C_{r} e^{i k y} \int_{-\infty}^{\sigma^{\prime}} e^{-\frac{\pi}{2} i u^{2}} d u\right) & \text { at } y<0 \\ \frac{1}{2}(1+i)\left(e^{-i k y} \int_{-\infty}^{\sigma} e^{-\frac{\pi}{2} i u^{2}} d u+e^{i k y} \int_{-\infty}^{\sigma^{\prime}} e^{-\frac{\pi}{2} i u^{2}} d u\right) & \text { at } y>0\end{cases}
$$

where

$$
\sigma^{2}=\frac{4}{\lambda}(r-y), \quad \sigma^{\prime 2}=\frac{4}{\lambda}(r+y), \lambda=\frac{2 \pi}{k}
$$

and $r=\sqrt{x^{2}+y^{2}}$. The signs of $\sigma$ and $\sigma^{\prime}$ in the $x-y$ plane are as shown in Fig. 2 .

\section{Fig. 2}

\subsection{Breakwater gap}

The plan view of a breakwater gap and the associated coordinate system are shown in Fig. 3. The origin of the horizontal coordinates is located at the center of the gap whose width is $B$. The solution is obtained by superposition from the solutions for the semi-infinite breakwaters located along the $x$-axis at $x>B / 2$ and $x<-B / 2$, respectively.

\section{Fig. 3}

To find the solution for the semi-infinite breakwater located at $x>B / 2$, we use the parameters:

$$
\begin{aligned}
& k\left(x-\frac{B}{2}\right)=\xi^{2}-\psi^{2}, k y=2 \xi \psi \\
& k r_{1}=\xi^{2}+\psi^{2}
\end{aligned}
$$

where $r_{1}$ is the distance of the point $(x, y)$ from the tip of the breakwater. The solution procedure is the same as that given in section 2.1. The solution is given as Eqs. (23) and (24) with 
$r_{1}$ in the place of $r$. Using Eqs. (25) and (26), $r_{1}$ is obtained as

$$
r_{1}=\sqrt{\left(x-\frac{B}{2}\right)^{2}+y^{2}}
$$

The signs of $\sigma$ and $\sigma^{\prime}$ in the $x-y$ plane are the same as shown in Fig. 2. Similarly, for the semi-infinite breakwater located at $x<-B / 2$, we use the parameters:

$$
\begin{aligned}
& k\left(x+\frac{B}{2}\right)=\xi^{2}-\psi^{2}, \quad k y=2 \xi \psi \\
& k r_{2}=\xi^{2}+\psi^{2}
\end{aligned}
$$

where $r_{2}$ is the distance of the point $(x, y)$ from the tip of the breakwater. Again, the solution is given as Eqs. (23) and (24) with $r_{2}$ in the place of $r$, and Eqs. (28) and (29) can be used to obtain $r_{2}$ as

$$
r_{2}=\sqrt{\left(x+\frac{B}{2}\right)^{2}+y^{2}}
$$

In this case, the signs of $\sigma$ and $\sigma^{\prime}$ are given as the symmetric transposition of Fig. 2 with respect to the $y$-axis.

The final solution is obtained by superposing the above two solutions for semi-infinite breakwaters. Because the signs of $\sigma$ and $\sigma^{\prime}$ are different between the two solutions, it is necessary to make them unified. For this, Eq. (23) for the semi-infinite breakwater located at $x>B / 2$ is changed to

$$
\begin{aligned}
& F(x, y)=e^{-i k \underline{y}} f_{1}+C_{r} g_{1} \quad \text { at } \quad x<\frac{B}{2}, y<0 \\
& =e^{-i k y}-f_{1}+g_{1} \quad \text { at } \quad x<\frac{B}{2}, y>0 \\
& =e^{-i k y}+C_{r} e^{i k y}-f_{1}-C_{r} g_{1} \quad \text { at } \quad x>\frac{B}{2}, \quad y<0
\end{aligned}
$$




$$
=f_{1}+g_{1} \quad \text { at } \quad x>\frac{B}{2}, y>0
$$

where

$$
\begin{aligned}
& f_{1}=\frac{1+i}{2} e^{-i k y} \int_{-\infty}^{\sigma_{1}} e^{-\frac{\pi}{2} i u^{2}} d u \\
& g_{1}=\frac{1+i}{2} e^{i k y} \int_{-\infty}^{\sigma_{1}^{\prime}} e^{-\frac{\pi}{2} i u^{2}} d u
\end{aligned}
$$

and

$$
\sigma_{1}^{2}=\frac{4}{\lambda}\left(r_{1}-y\right), \quad \sigma_{1}^{\prime 2}=\frac{4}{\lambda}\left(r_{1}+y\right)
$$

Now $\sigma_{1}$ and $\sigma_{1}{ }^{\prime}$ have minus signs in the whole domain. The solution for the semi-infinite breakwater located at $x<-B / 2$ can also be modified similarly. Finally, the superposition of the two solutions yields the solution for wave scattering by a breakwater gap as

$$
\begin{aligned}
F(x, y) & =e^{-i k y}+C_{r} e^{i k y}-f_{1}+C_{r} g_{1}-f_{2}-C_{r} g_{2} & & \text { at } x<-\frac{B}{2}, y<0 \\
& =-f_{1}+g_{1}+f_{2}+g_{2} & & \text { at } x<-\frac{B}{2}, y>0 \\
& =e^{-i k y}-f_{1}+C_{r} g_{1}-f_{2}+C_{r} g_{2} & & \text { at }-\frac{B}{2}<x<\frac{B}{2}, y<0 \\
& =e^{-i k y}-f_{1}+g_{1}-f_{2}+g_{2} & & \text { at }-\frac{B}{2}<x<\frac{B}{2}, y>0 \\
& =e^{-i k y}+C_{r} e^{i k y}-f_{1}-C_{r} g_{1}-f_{2}+C_{r} g_{2} & & \text { at } x>\frac{B}{2}, y<0 \\
& =f_{1}+g_{1}-f_{2}+g_{2} & & \text { at } x>\frac{B}{2}, y>0
\end{aligned}
$$

where 


$$
\begin{aligned}
& f_{2}=\frac{1+i}{2} e^{-i k y} \int_{-\infty}^{\sigma_{2}} e^{-\frac{\pi}{2} i u^{2}} d u \\
& g_{2}=\frac{1+i}{2} e^{i k y} \int_{-\infty}^{\sigma_{2}^{\prime}} e^{-\frac{\pi}{2} i u^{2}} d u
\end{aligned}
$$

and

$$
\sigma_{2}^{2}=\frac{4}{\lambda}\left(r_{2}-y\right), \quad \sigma_{2}^{\prime 2}=\frac{4}{\lambda}\left(r_{2}+y\right)
$$

Again $\sigma_{2}$ and $\sigma_{2}{ }^{\prime}$ have minus signs in the whole domain.

\section{Results}

Fig. 4 shows diffraction coefficients (i.e. wave amplitude relative to the incident amplitude) around a semi-infinite breakwater in the cases of $C_{r}=1.0$ and 0.5 . The results are presented in terms of dimensionless coordinates, $x / L$ and $y / L$, where $L$ is the wavelength. The solution of $C_{r}=1.0$ becomes the solution of Penney and Price (1952). As expected, the diffraction coefficient behind the breakwater is not affected by the reflection coefficient of the breakwater. In the sea side of the breakwater, however, in addition to the decrease of standing wave height in front of the breakwater $(x \geq 0)$, the area of larger wave height decreases in the open area $(x<0)$, as the reflection coefficient decreases.

Fig. 5 shows a comparison of diffraction coefficients at $y=-4 L$ between $C_{r}=1.0$ and 0.5. As the reflection coefficient decreases from 1.0 to 0.5 , the reflected wave height reduces to a half so that the diffraction coefficient decreases from about 2.0 to 1.5 in front of the breakwater. The diffraction coefficient shows less variation for smaller reflection coefficient in the open area $(x<0)$ as well.

Fig. 6 shows diffraction coefficients around a breakwater gap of width $B=2 L$ in the cases of $C_{r}=1.0$ and 0.5. Again, as expected, the diffraction coefficient behind the gap is not affected by the reflection coefficient of the breakwater. In the seaward side of the gap, however, in addition to the decrease of standing wave height in front of the breakwaters $(x / L<-1$ and $x / L>1)$, the agitation in front of the gap $(-1 \leq x / L \leq 1)$ also reduces, as the reflection coefficient decreases.

Fig. 7 shows a comparison of diffraction coefficients at $y=-4 L$ between $C_{r}=1.0$ and 0.5 . 
As the reflection coefficient decreases from 1.0 to 0.5 , the reflected wave height reduces to a half so that the diffraction coefficient decreases from about 2.0 to 1.5 in front of the breakwaters. The diffraction coefficient in front of the gap $(-1 \leq x / L \leq 1)$ also reduces a little with smaller reflection coefficient.

\begin{tabular}{|c|}
\hline Fig. 4 \\
\hline Fig. 5 \\
\hline Fig. 6 \\
\hline Fig. 7 \\
\hline
\end{tabular}

To examine the effect of reflection coefficient upon the tranquility of harbor entrance, the diffraction coefficients for different reflection coefficients are plotted in Fig. 8 along three transects of $x / L=0,-1$, and -2 in the case of a semi-infinite breakwater (cf. Fig. 1). First, it can be seen that the diffraction coefficient significantly reduces as departing from the tip of the breakwater. The difference between the maximum and minimum of diffraction coefficient decreases as the reflection coefficient decreases. In the transect passing the tip of the breakwater ( $x / L=0)$, the maximum diffraction coefficient decreases from about 1.45 to 1.2 as the reflection coefficient decreases from 1.0 to 0.5 . In the area far from the breakwater, though the diffraction coefficient itself is small, the relative effect of reflection coefficient is still significant. For example, at the transect of $x / L=-2$, the maximum diffraction coefficient decreases from about 1.1 to 1.04 as the reflection coefficient decreases from 1.0 to 0.5 .

Fig. 9 shows the variation of diffraction coefficients for different reflection coefficients along transects of $x / L=0$ and \pm 1 in the case of a breakwater gap (cf. Fig. 2). In the transect passing the center of the gap ( $x / L=0)$, the maximum diffraction coefficient increases as going away from the gap because the waves reflected from the breakwater diffract toward the area in front of the gap. On the other hand, in the transect passing the tip of the breakwater $(x / L= \pm 1)$, the maximum diffraction coefficient increases with the distance from the breakwater in the area near the breakwater, and it decreases when $y / L<-2$ and increases again when $y / L<-5$. As with the semi-infinite breakwater, the difference between the maximum and minimum of diffraction coefficient decreases as the reflection coefficient decreases. In the transect passing the center of the gap $(x / L=0)$, the maximum diffraction coefficient decreases from about 1.5 to 1.2 at $y / L \approx-6$ as the reflection coefficient decreases from 1.0 to 0.5 . The same can be seen at $y / L \approx-2$ of the transect passing the tip of the breakwater $(x / L= \pm 1)$. 


\section{Conclusions}

In this study, we have derived analytic solutions for water wave scattering by a semi-infinite breakwater or a breakwater gap of partial reflection. The derived solution has been used to examine the effect of the reflection coefficient at the front face of the breakwater upon the wave climate near the breakwater or gap. It has been shown that the reduced reflection coefficient not only reduces the standing wave height in front of the breakwater but also reduces wave agitation at the entrance of a harbor.

In this study, it was assumed that the front face of the breakwater is of partial reflection while the back face is of perfect reflection. However, the boundary condition at the back face of the breakwater would not affect the solution because the wave height is essentially zero there and also the waves propagate parallel to the breakwater. Therefore, the present solution could be used for a breakwater of arbitrary reflection coefficients at both front and back faces. The present solution is limited to normally incident waves. An extension to oblique incidence may be necessary.

\section{Acknowledgements}

The writers were supported by the Korea Sea Grant Program and Brain Korea 21 Project. The first writer was also supported by the Project for Development of Reliability Based Design Method for Port and Harbor Structures of Korea Ministry of Marine Affairs and Fisheries.

\section{References}

Kim, H. (2007). Wave scattering by a semi-infinite breakwater or a breakwater gap with porous front and solid back, Master thesis, Seoul National University, Seoul, Korea.

Lamb, H. (1945). Hydrodynamics, 6th ed., Dover, New York.

McBride, M.W., Smallman, J.V., and Allsop, N.W.H. (1994). Design of harbour entrances: Breakwater design and vessel safety. Proc. Int. Conf. on Hydro-Technical Engineering for Port and Harbor Construction, PHRI, Yokosuka, Japan, pp. 525-541.

McIver, P. (1999). Water-wave diffraction by thin porous breakwater. Journal of Waterway, Port, Coastal 
and Ocean Engineering, Vol. 125, No. 2, pp. 66-70.

Penney, W.G. and Price, A.T. (1952). The diffraction theory of sea waves and the shelter afforded by breakwaters. Philosophical Transactions of the Royal Society, Series A, Vol. 244, pp. 236253.

Sollitt, C.K. and Cross, R.H. (1972). Wave transmission through porous breakwaters. Proc.13th Int. Conf. on Coastal Engineering, ASCE, Vancouver, Canada, pp. 1827-1846.

Sommerfeld, A. (1896). Mathematische theorie der diffraction. Mathematische Annalen, Vol. 47, pp. 317-374.

Yu, X. (1995). Diffraction of water waves by porous breakwaters. Journal of Waterway, Port, Coastal and Ocean Engineering, Vol. 121, No. 6, pp. 275-282. 
(1)

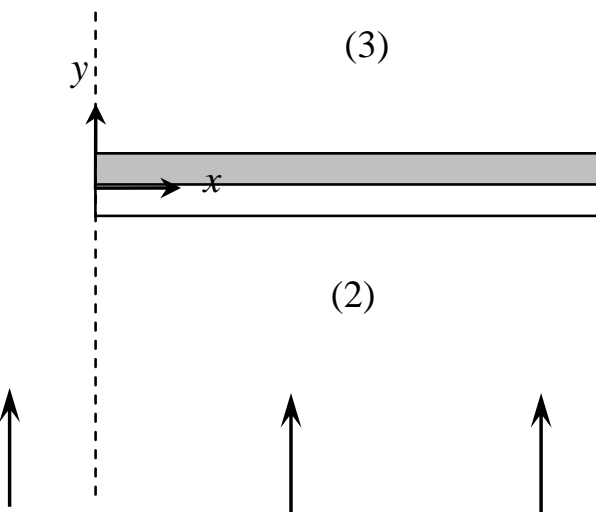

Figure 1. Definition sketch of a semi-infinite breakwater 


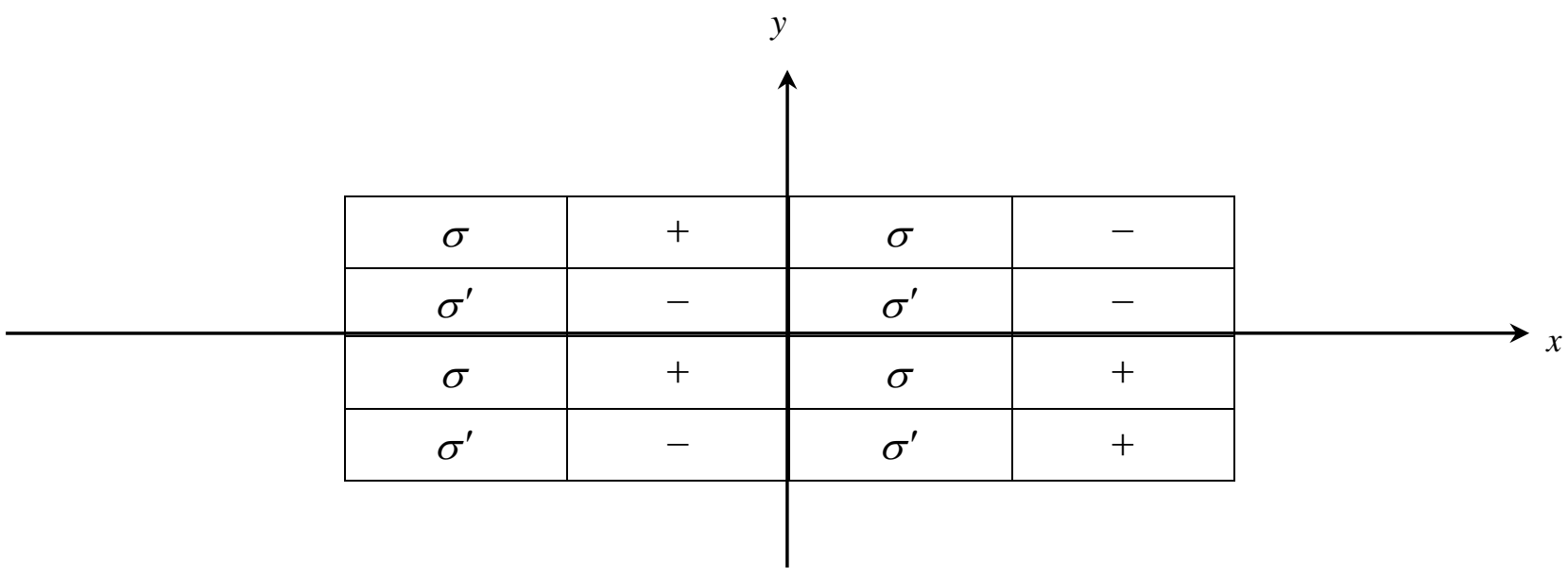

Figure 2. Signs of $\sigma$ and $\sigma^{\prime}$ for a semi-infinite breakwater located at $y=0, x>0$ 


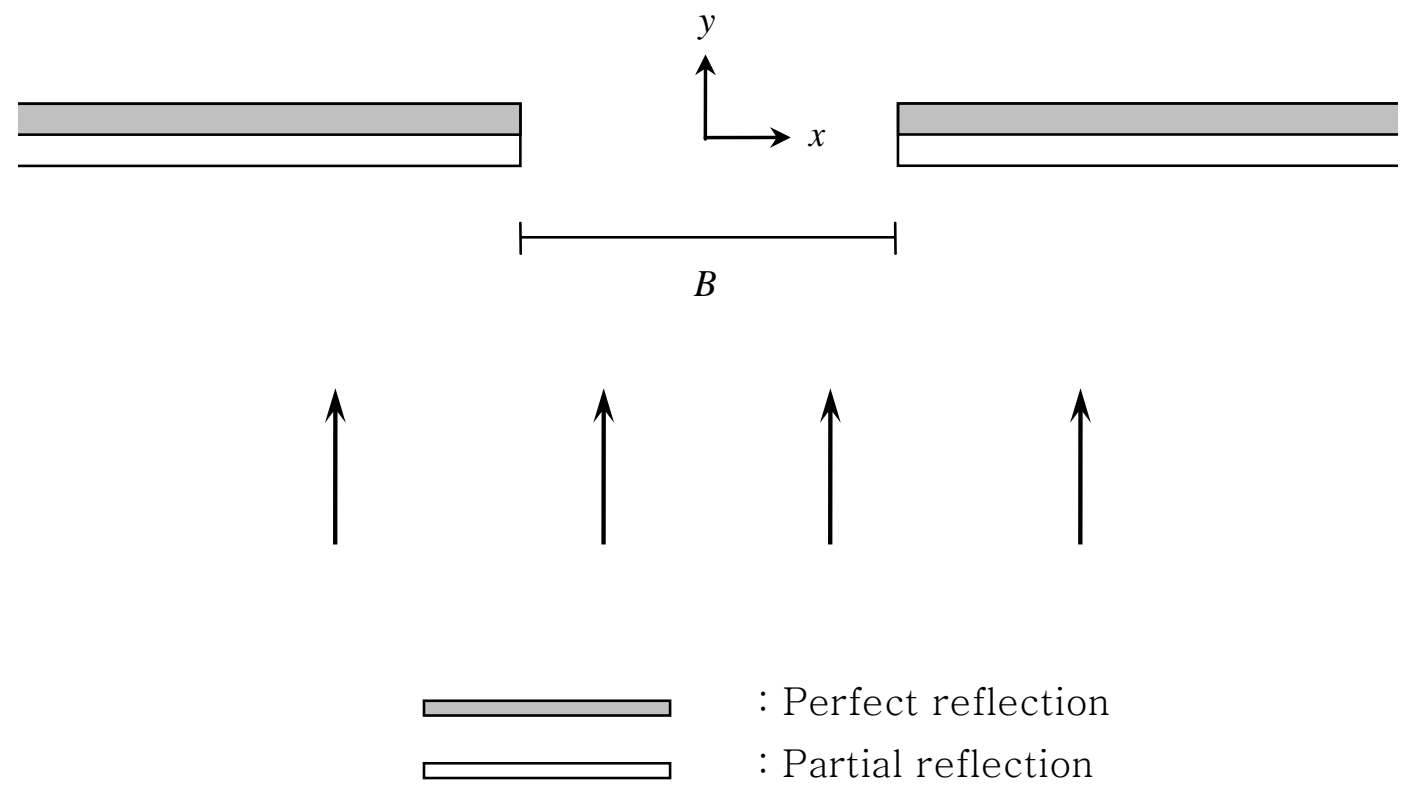

Figure 3. Definition sketch of a breakwater gap 


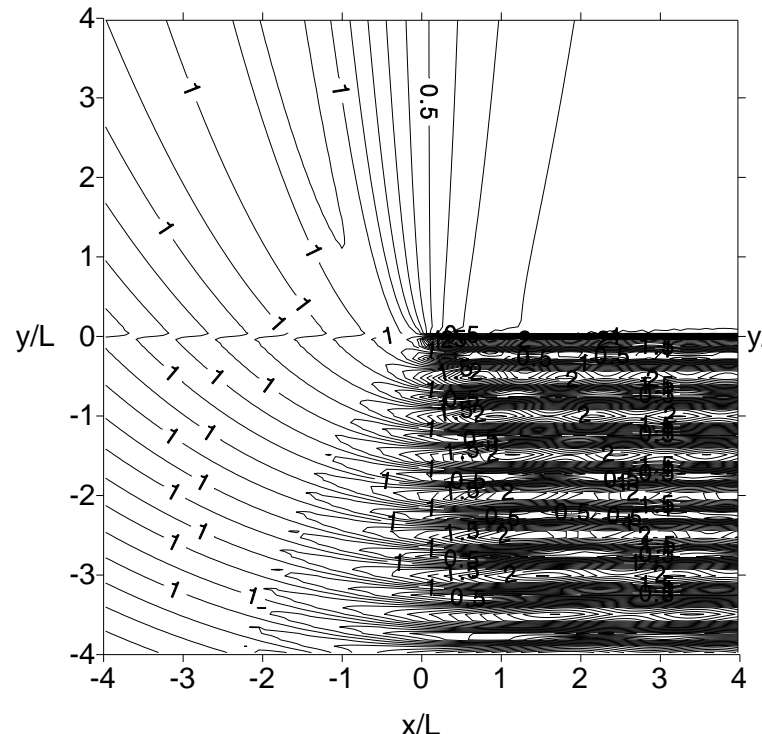

(a)

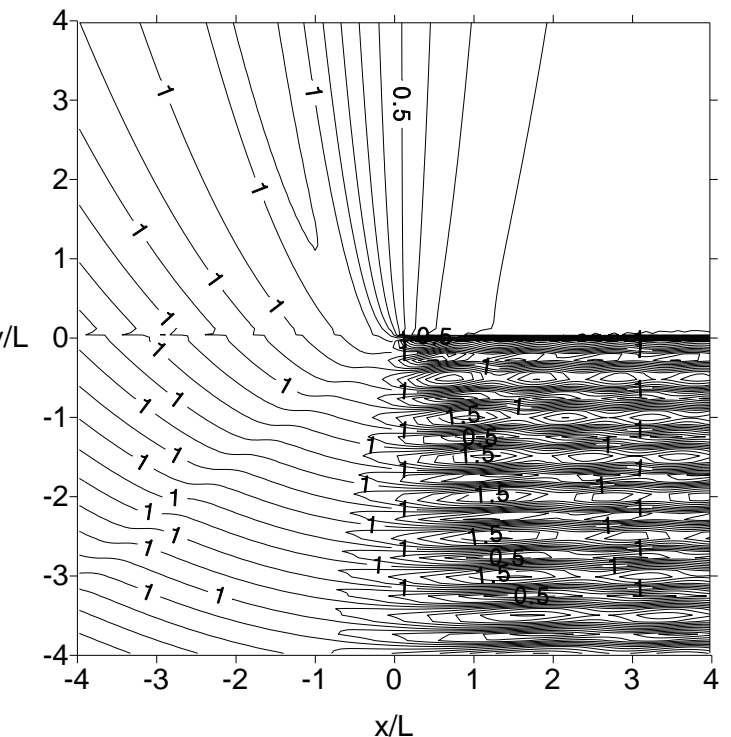

(b)

Figure 4. Contours of diffraction coefficients in the vicinity of semi-infinite breakwater: (a) $C_{r}=1.0$; (b) $C_{r}=0.5$ 


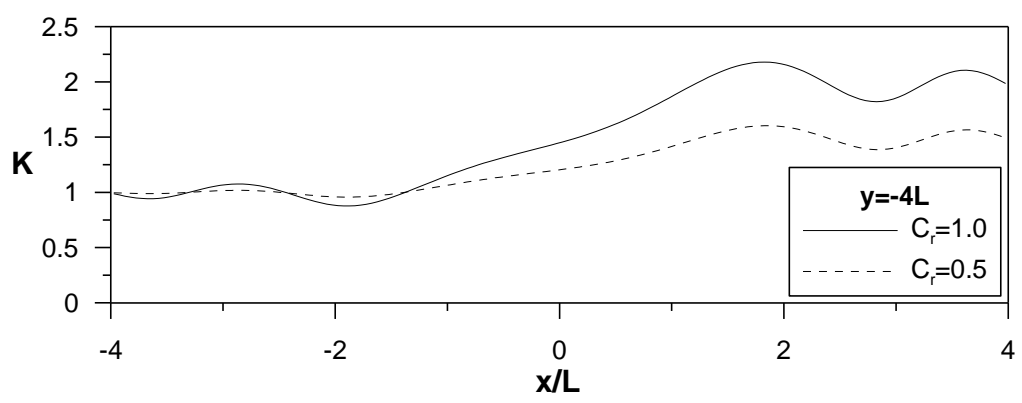

Figure 5. Comparison of diffraction coefficients for different reflection coefficients at $y=-4 L$ of a semi-infinite breakwater 


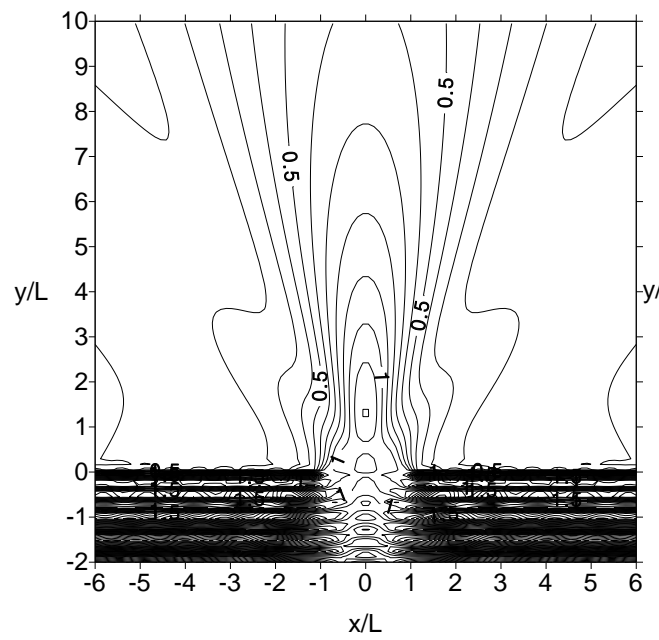

(a)

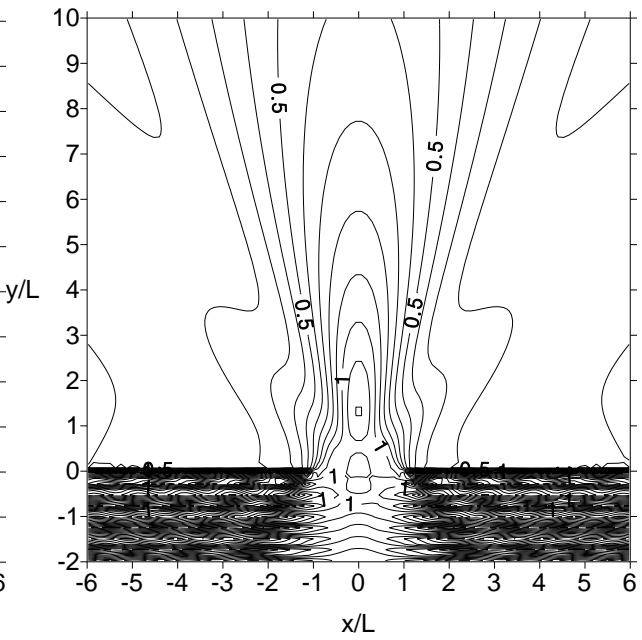

(b)

Figure 6. Contours of diffraction coefficients in the vicinity of a breakwater gap: (a) $C_{r}=1.0$; (b) $C_{r}=0.5$ 


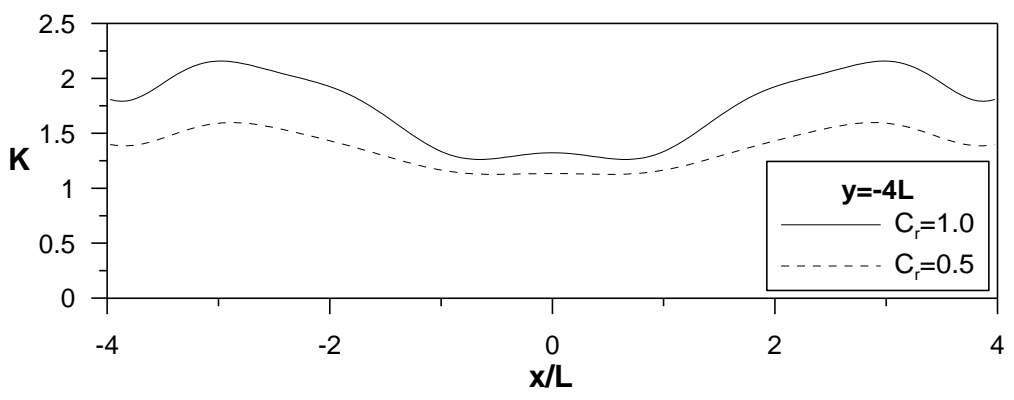

Figure 7. Comparison of diffraction coefficients for different reflection coefficients at $y=-4 L$ of a breakwater gap 


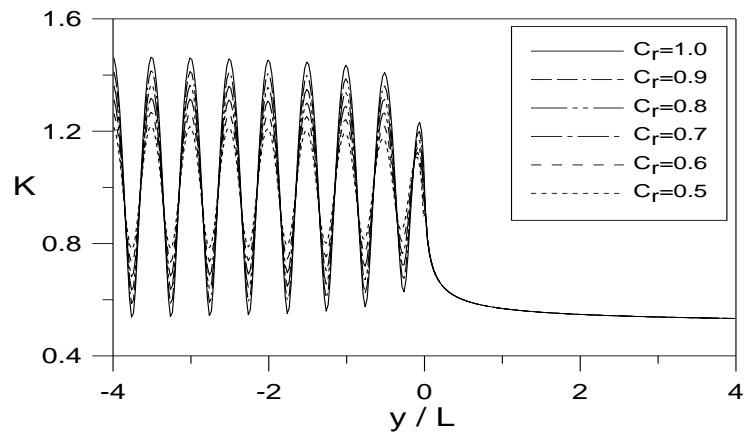

(a)

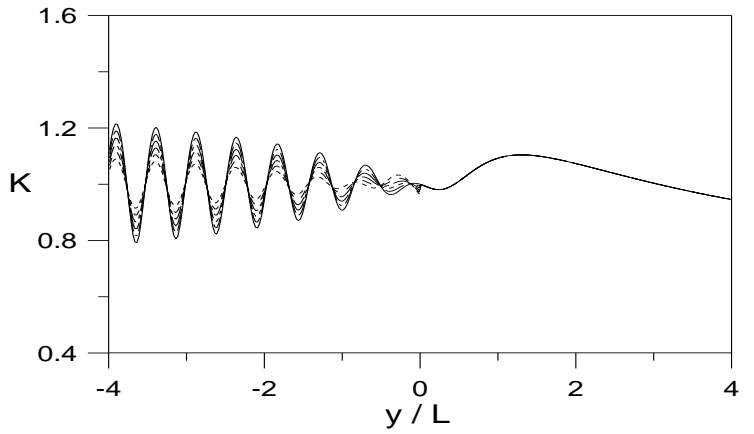

(b)

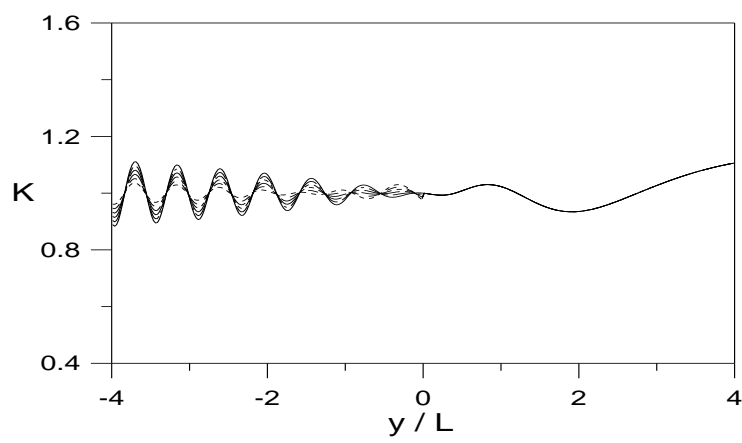

(c)

Figure 8. Comparison of diffraction coefficients along different transects parallel to $y$-axis for semi-infinite breakwater with different reflection coefficients: (a) $x / L=0$; (b) $x / L=-1$; (c) $x / L=-2$ 


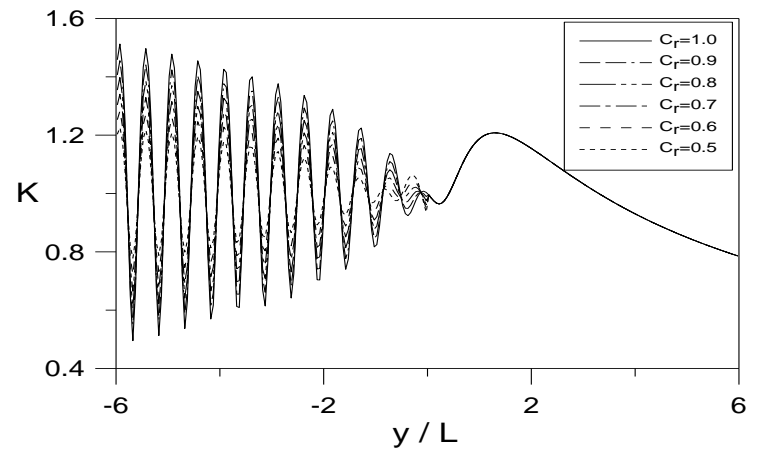

(a)

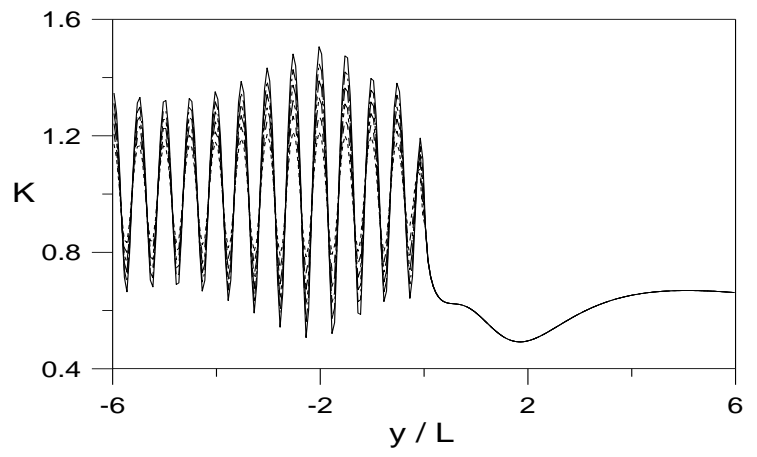

(b)

Figure 9. Comparison of diffraction coefficients along different transects parallel to $y$-axis for breakwater gap of $B=2 L$ with different reflection coefficients: (a) $x / L=0$; (b) $x / L= \pm 1$ 